\title{
Hybrid Combinatorial Optimization: Sample Problems and Algorithms
}

Vaibhav Srivastava

\author{
Francesco Bullo
}

\begin{abstract}
We study a class of non-convex optimization problems involving sigmoid functions. We show that sigmoid functions impart a combinatorial element to the optimization variables and make them hybrid of continuous and discrete variables. We formulate versions of the knapsack problem and the bin-packing problem with such hybrid variables. We utilize the approximation algorithms from the combinatorial optimization literature and develop approximation algorithms for these NP-hard hybrid optimization problems.
\end{abstract}

\section{INTRODUCTION}

The modern times have witnessed extensive deployment of camera networks for surveillance. The feeds from these camera networks are send to a central location, where a human operator looks at them to decide on the presence of some malicious activity [5]. The plethora of information available from these feeds results in information overload and is often the root cause for missing critical information [17]. This calls for an investigation into the optimal policies to handle this information overload.

Recent advances in cognitive psychology [2] have shown that the performance of a human operator in a decision making task evolves as a sigmoid function of the time she allocates to it. This performance of the human operator should be accounted in order to develop optimal policies for aforementioned human in the loop systems.

In this paper we study certain non-convex resource allocation problems with sigmoid utilities. We present versions of the knapsack problem and the bin-packing problem where each item has a sigmoid utility. If the utilities are step functions, then these problems reduce to standard knapsack and bin-packing problems [13]. Similarly, if the utilities were concave functions then these problems reduce to standard convex resource allocation problems [10]. We will show that with sigmoid utilities the optimization problem becomes a hybrid of combinatorial optimization problem and convex resource allocation problem.

The knapsack problem [12] has been extensively studied. A considerable emphasis has been on the combinatorial knapsack problem [13] and the knapsack problems with concave utilities. A survey is presented in [4]. Certain knapsack problems with piecewise linear utilities have been studied in [11]. Moré et al [15] and Burke et al [6] study knapsack problem with convex utilities.

Recently, the optimization problems with sigmoid utilities have received a significant attention. Fazel et al [7] study

This work has been supported in part by AFOSR MURI Award-FA955007-1-0528 and NSF Award CPS-1035917.

The authors thank Dr. Cédric Langbort for insightful discussions.

Vaibhav Srivastava and Francesco Bullo are with Center for Control, Dynamical Systems, and Computation, University of California, Santa Barbara, Santa Barbara, CA 93106, USA, \{vaibhav, bullo\} eengineering.ucsb.edu network utility maximization problem where utility of each source is a sigmoid function of the flow through it. They utilize sum-of-squares relaxation to determine an approximate solution to this problem. Certain optimal servicing policies for a queue with sigmoid performance of the operator are presented in [18]. Ginsberg [9] study a knapsack problem where each item has identical sigmoid utility. Freeland et al [8] discuss the implication of sigmoid functions on decision models and present an approximation algorithm for the knapsack problem with sigmoid utilities that constructs a concave envelop of the sigmoid functions and thus, solves the resulting convex problem. Ağrali et al [1] consider the same knapsack problem and show that this problem is NP-hard. They relax the problem by constructing a concave envelop of the sigmoid function and then determine the global optimal solution using branch and bound techniques.

We study optimization problems with sigmoid functions. We show that sigmoid utility renders a combinatorial element to the problem and resource allocated to each item under optimal policy is either zero or more than a critical value. Thus, optimization variable has both continuous and discrete features. We refer to such variables by hybrid variables and an optimization problem involving such variables by hybrid optimization problem. We present hybrid versions of the knapsack problem and the bin-packing problem. In particular, we study the following problems: First, given a set of items with sigmoid utilities and a fixed resource, determine the optimal resource allocation to each item. Second, given a set of items with sigmoid utilities and an unlimited number of bins with fixed resource available at each bin, determine the minimum number of bins and a mapping of each item to some bin, such that optimal allocation in the first problem allocates non-zero resource to each item in every bin. These problems model situations where a human operator is looking at the feeds from a camera network and deciding on the presence of some malicious activity. The first problem determines the optimal fraction of work-hours, the operator should allocate to each feed such that her overall performance is optimal. Assuming that the operators work in an optimal fashion, the second problem determines the minimum number of operators and an allocation of each feed to some operator such that each operator allocates non-zero fraction of work-hours to each feed assigned to her. The major contributions of this work are:

i) We show that the optimization problems with sigmoid functions involve hybrid variables.

ii) We formulate hybrid versions of the knapsack problem and the bin-packing problem.

iii) We utilize the algorithms from the combinatorial optimization to develop algorithms for these hybrid opti- 
mization problems.

iv) We develop constant factor approximation algorithms for these hybrid optimization problems.

The remainder of the paper is organized in the following way. We present some preliminaries in Section II. Implications of the sigmoid functions on the optimal policies are discussed in Section III. We formulate and solve the hybrid knapsack problem in Section IV. The hybrid binpacking problem is treated in Section V. Our conclusions are presented in Section VI.

\section{PREliminaries}

\section{A. Speed-accuracy trade-off in human decision making}

Consider the scenario where, based on the collected evidence, the human has to decide on one of the two alternatives $H_{0}$ and $H_{1}$. The evolution of the probability of correct decision in such scenarios has been studied in cognitive psychology literature [16], [2] and following models have been proposed:

Pew's model: The probability of deciding on hypothesis $H_{1}$, given that hypothesis $H_{1}$ is true, at a given time $t \in \mathbb{R}_{\geq 0}$ is given by

$$
\mathbb{P}\left(\text { say } H_{1} \mid H_{1}, t\right)=\frac{p_{0}}{1+e^{-(a t-b)}},
$$

where $p_{0} \in[0,1], a, b \in \mathbb{R}$ are some parameters which depend on the human operator [16].

Drift diffusion model: Conditioned on the hypothesis $H_{1}$, the evolution of the evidence for decision is modeled as a drift-diffusion process [2]. Given a drift rate $\beta>0$, and diffusion rate $\sigma$, with a decision threshold $\nu$, the conditional probability of the correct decision is

$$
\mathbb{P}\left(\text { say } H_{1} \mid H_{1}, t\right)=\frac{1}{\sqrt{2 \pi \sigma^{2} t}} \int_{\nu}^{\infty} e^{\frac{-(\Lambda-\beta t)^{2}}{2 \sigma^{2} t}} d \Lambda,
$$

where $\Lambda \equiv \mathcal{N}\left(\beta t, \sigma^{2} t\right)$ is the evidence at time $t$.

\section{B. Sigmoid functions}

A smooth function $f: \mathbb{R}_{\geq 0} \rightarrow \mathbb{R}_{\geq 0}$ defined by

$$
f(t)=f_{\mathrm{cvx}}(t) \mathbf{1}\left(t<t^{\mathrm{inf}}\right)+f_{\mathrm{cnv}}(t) \mathbf{1}\left(t \geq t^{\mathrm{inf}}\right),
$$

where $f_{\mathrm{cvx}}$ and $f_{\mathrm{cnv}}$ are monotonically increasing convex and concave functions, respectively, $\mathbf{1}(\cdot)$ is the indicator function, and $t^{\text {inf }}$ is the inflection point. Derivative of sigmoid function is unimodal with maximum at $t^{\text {inf }}$. Further, $f^{\prime}(0) \geq 0$ and $\lim _{t \rightarrow \infty} f^{\prime}(t)=0$. A typical graph of a sigmoid function and its derivative is shown in Figure 1. Note that the evolution of the conditional probabilities of correct decision are sigmoid functions in Pew's as well as drift-diffusion model.

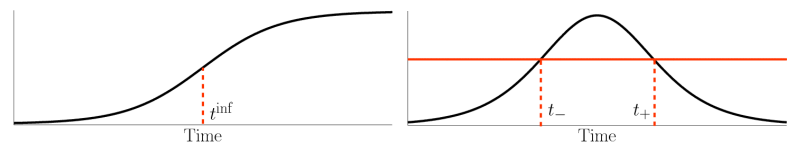

Fig. 1. A typical sigmoid function and its derivative.

\section{Knapsack problem}

Given $N$ items with values $v_{i}$ and costs $c_{i}, i \in\{1, \ldots, N\}$, the knapsack problem is to pick a set of items such that the value of picked items is maximized for a given total cost $C$. Formally, the knapsack problem [13], [12] is posed as:

$$
\begin{array}{cl}
\underset{\boldsymbol{x}}{\operatorname{maximize}} & \sum_{i=1}^{N} v_{i} x_{i} \\
\text { subject to } & \sum_{i=1}^{N} c_{i} x_{i} \leq C \\
& x_{i} \in\{0,1\} .
\end{array}
$$

The knapsack problem is NP-hard [13]. A 2-factor approximation algorithm that runs in $O(N)$ time is presented in Algorithm 1.

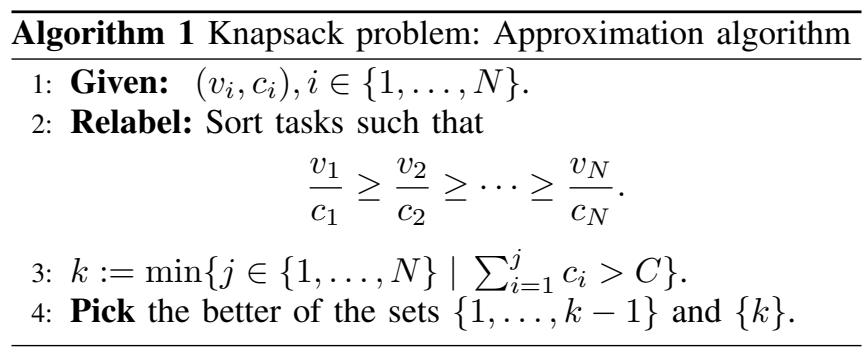

\section{Bin-packing Problem}

Given a set of items with size $\left\{a_{i}<1\right\}_{i \in\{1, \ldots, N\}}$, and identical bins of unit size. The bin-packing problem [13] is to determine the assignment of each item $\Upsilon:\{1, \ldots, N\} \rightarrow$ $\{1, \ldots, K\}$ such that the number of bins utilized $K$ is minimum and the items allocated to each bin can be packed into it. Formally, the bin-packing problem is stated as following:

$$
\begin{array}{ll}
\text { minimize } & K \\
\text { subject to } & \sum_{i \in \mathcal{A}_{j}} a_{i} \leq 1, \quad \forall j \in\{1, \ldots, K\}
\end{array}
$$

where $\mathcal{A}_{j}=\{\ell \in\{1, \ldots, N\} \mid \Upsilon(\ell)=j\}$.

The bin-packing problem is strongly $N P$ complete and for any $\rho<3 / 2$, there exists no $\rho$-factor approximation scheme for it, unless $P=N P$. The next fit algorithm is a 2 -factor approximation scheme for the bin-packing problem and runs in $O(N)$ time [13]. It is presented in Algorithm 2.

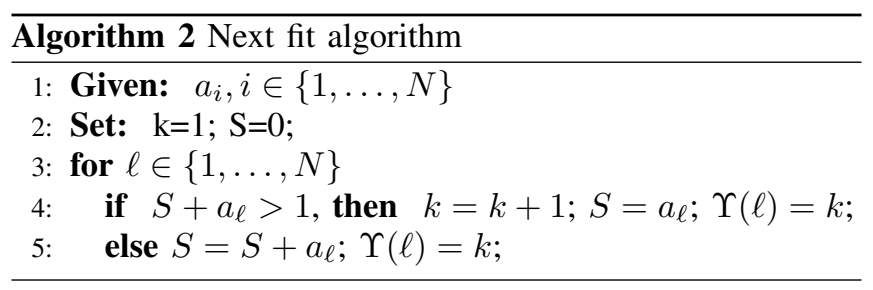

\section{Sigmoid Function And Linear Penalty}

In order to gain insight into the behavior of sigmoid functions, we start with a simple problem with a very interesting result. We study the maximization of a sigmoid function subject to a linear penalty. In particular, given a sigmoid function $f$ and a penalty rate $c \in \mathbb{R}_{>0}$, we wish to solve the following problem:

$$
\underset{t \geq 0}{\operatorname{maximize}} f(t)-c t
$$

The derivative of a sigmoid function is not a one to one mapping and hence, not invertible. We define the pseudoinverse of the derivative of a sigmoid function $f$ with inflection point $t^{\text {inf }}, f^{\dagger}: \mathbb{R}_{>0} \rightarrow \mathbb{R}_{\geq 0}$ by 
$f^{\dagger}(y)= \begin{cases}\max \left\{t \in \mathbb{R}_{\geq 0} \mid f^{\prime}(t)=y\right\}, & \left.\text { if } y \in] 0, f^{\prime}\left(t^{\mathrm{inf}}\right)\right], \\ 0, & \text { otherwise. }\end{cases}$

We now present the solution to the problem (1).

Lemma 1 (Sigmoid function with linear penalty): For the optimization problem (1), the optimal allocation $t^{*}$ is

$$
t^{*}:=\operatorname{argmax}\left\{f(\beta)-c \beta \mid \beta \in\left\{0, f^{\dagger}(c)\right\}\right\} .
$$

Proof: The global maximum lies at the point where first derivative is zero or at the boundary. The first derivative of the objective function is $f^{\prime}(t)-c$. If $f^{\prime}\left(t^{\text {inf }}\right)<c$, then the objective function is a decreasing function of time and the maximum is achieved at $t^{*}=0$. Otherwise, a critical point is obtained by setting first derivative zero. We note that $f^{\prime}(t)=c$ has at most two roots. It can be verified using the second derivative conditions that if there exist two roots, then the bigger of the two roots corresponds to a local maximum. Otherwise, the only root corresponds to a local maximum. The global maximum is determined by comparing the local maximum with the value of the objective function at the boundary $t=0$. This completes the proof.

The optimal solution to problem (1) for different values of penalty rate $c$ is shown in Figure 2. One may notice the optimal allocation jumps down to zero at a critical penalty rate. This jump in the optimal allocation gives rise to combinatorial effects in the problems involving multiple sigmoid functions.

Definition 2 (Critical penalty rate): Given a sigmoid function $f$ and linear penalty, we refer to the maximum penalty rate at which problem (1) has a non-zero solution by critical penalty rate. Formally, for a given sigmoid function $f$ and penalty rate $c \in \mathbb{R}_{>0}$, let the solution of the problem (1) be $t_{f, c}^{*}$, the critical penalty rate $\psi_{f}$ is defined by

$$
\psi_{f}=\max \left\{c \in \mathbb{R}_{>0} \mid t_{f, c}^{*} \in \mathbb{R}_{>0}\right\} .
$$

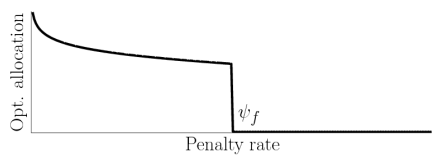

Fig. 2. Optimal allocation to a sigmoid function as a function of linear penalty

\section{Hybrid KNAPSACK PRoblem}

\section{A. Problem description}

Given sigmoid functions $f_{\ell}$ with associated weights $w_{\ell}, \ell \in\{1, \ldots, N\}$, and resource $T \in \mathbb{R}_{>0}$, the hybrid knapsack problem is to determine $\boldsymbol{t} \in \mathbb{R}_{\geq 0}^{N}$ that solves the following optimization problem:

$$
\begin{array}{cl}
\underset{\boldsymbol{t}}{\operatorname{maximize}} & \sum_{\ell=1}^{N} w_{\ell} f_{\ell}\left(t_{\ell}\right) \\
\text { subject to } & \sum_{\ell=1}^{N} t_{\ell} \leq T \\
& \boldsymbol{t} \succeq 0 .
\end{array}
$$

The hybrid knapsack problem models the situation where a human operator has to perform $N$ decision making tasks within time $T$. If the performance of the human operator on task $\ell$ is given by sigmoid function $f_{\ell}$ and a weight $w_{\ell}$ is assigned to it, then the optimal duration allocation to each task is determined by the solution of the hybrid knapsack problem (3).

\section{B. Optimal solution}

Before we determine the solution to the hybrid knapsack problem, we introduce some notations. We define the Lagrangian $L: \mathbb{R}_{>0}^{N} \times \mathbb{R}_{\geq 0} \times \mathbb{R}_{\geq 0}^{N} \rightarrow \mathbb{R}$ for the hybrid knapsack problem (3) by

$$
L(\boldsymbol{t}, \alpha, \boldsymbol{\mu})=\sum_{\ell=1}^{N} w_{\ell} f_{\ell}\left(t_{\ell}\right)+\alpha\left(T-\sum_{\ell=1}^{N} t_{\ell}\right)+\boldsymbol{\mu}^{\mathrm{T}} \boldsymbol{t} .
$$

Let $t_{\ell}^{\text {inf }}$ be the inflection point of sigmoid function $f_{\ell}$ and $f_{\ell}^{\dagger}$ be the pseudo-inverse of its derivative as defined in equation (2). We define the maximum weighted derivative of sigmoid function $f_{\ell}$ by $\alpha_{\ell}=w_{\ell} f_{\ell}^{\prime}\left(t_{\ell}^{\mathrm{inf}}\right)$. We also define $\alpha_{\max }=\max \left\{\alpha_{\ell} \mid \ell \in\{1, \ldots, N\}\right\}$. We will later show that $\alpha_{\max }$ is the maximum possible value of the Lagrange multiplier $\alpha$.

We define the set of inconsistent sigmoid functions by $\mathcal{I}=\left\{\ell \in\{1, \ldots, N\} \mid t_{\ell}^{\text {inf }}>T\right\}$. We denote the $j^{\text {th }}$ element of the standard basis of the real coordinate space by $\boldsymbol{e}_{j}$.

Define $\left.F:] 0, \alpha_{\max }\right] \rightarrow \mathbb{R}_{\geq 0}$ as the optimal value of the objective function in the following $\alpha$-parametrized knapsack problem:

$$
\begin{array}{ll}
\operatorname{maximize} & \sum_{\ell=1}^{N} x_{\ell} w_{\ell} f_{\ell}\left(f_{\ell}^{\dagger}\left(\alpha / w_{\ell}\right)\right) \\
\text { subject to } & \sum_{\ell=1}^{N} x_{\ell} f_{\ell}^{\dagger}\left(\alpha / w_{\ell}\right) \leq T \\
& x_{\ell} \in\{0,1\}, \quad \forall \ell \in\{1, \ldots, N\} .
\end{array}
$$

Let $\left.\left.F_{\text {approx }}:\right] 0, \alpha_{\max }\right] \rightarrow \mathbb{R}_{\geq 0}$ be the approximate optimal value of the objective function in the $\alpha$-parametrized knapsack problem obtained through Algorithm 1. Define $\left.\left.F_{\mathrm{LP}}:\right] 0, \alpha_{\max }\right] \rightarrow \mathbb{R}_{\geq 0}$ as the optimal value of the objective function in the following $\alpha$-parametrized fractional knapsack problem:

$$
\begin{array}{cl}
\operatorname{maximize} & \sum_{\ell=1}^{N} x_{\ell} w_{\ell} f_{\ell}\left(f_{\ell}^{\dagger}\left(\alpha / w_{\ell}\right)\right) \\
\text { subject to } & \sum_{\ell=1}^{N} x_{\ell} f_{\ell}^{\dagger}\left(\alpha / w_{\ell}\right) \leq T \\
& x_{\ell} \in[0,1], \quad \forall \ell \in\{1, \ldots, N\} .
\end{array}
$$

Before we state approximation algorithm to solve hybrid knapsack problem, we prove the following important property of the function $F_{\mathrm{LP}}$.

Lemma 3 (Discontinuity of $F_{\mathrm{LP}}$ ): The maximal set of points of discontinuity of the function $F_{\mathrm{LP}}$ is $\left\{\alpha_{1}, \ldots, \alpha_{N}\right\}$.

Proof: For each $\alpha \in\left[0, \alpha_{\max }\right]$, the $\alpha$-parametrized fractional knapsack problem is a linear program, and the solution lies at one of the vertex of the feasible simplex. Note that if $f_{\ell}^{\dagger}\left(\alpha / w_{\ell}\right)$ is a continuous function for each $\ell \in\{1, \ldots, N\}$, then the vertices of the feasible simplex are continuous functions of $\alpha$. Further, the objective function is also continuous if $f_{\ell}^{\dagger}\left(\alpha / w_{\ell}\right)$ is a continuous function for each $\ell \in\{1, \ldots, N\}$. Therefore, the function $F_{\mathrm{LP}}$ may be 
discontinuous only if $f_{\ell}^{\dagger}\left(\alpha / w_{\ell}\right)$ is discontinuous for some $\ell$, i.e., $\alpha \in\left\{\alpha_{1}, \ldots, \alpha_{N}\right\}$.

We will show that if all the sigmoid functions are consistent, then the allocation to each sigmoid function can be written in terms of the Lagrange multiplier $\alpha$, and the hybrid knapsack problem (3) reduces to $\alpha$-parametrized knapsack problem (4). Further, the optimal Lagrange multiplier $\alpha^{*}$ can be searched in the interval $\left.] 0, \alpha_{\max }\right]$. In order to determine a constant factor polynomial time approximation algorithm, we approximately solve the $\alpha$-parametrized knapsack problem in Algorithm 3. This algorithm also involves a performance improvement heuristic where unemployed resource is allocated to the most beneficial sigmoid function amongst those with zero resource. We now analyze the algorithm.

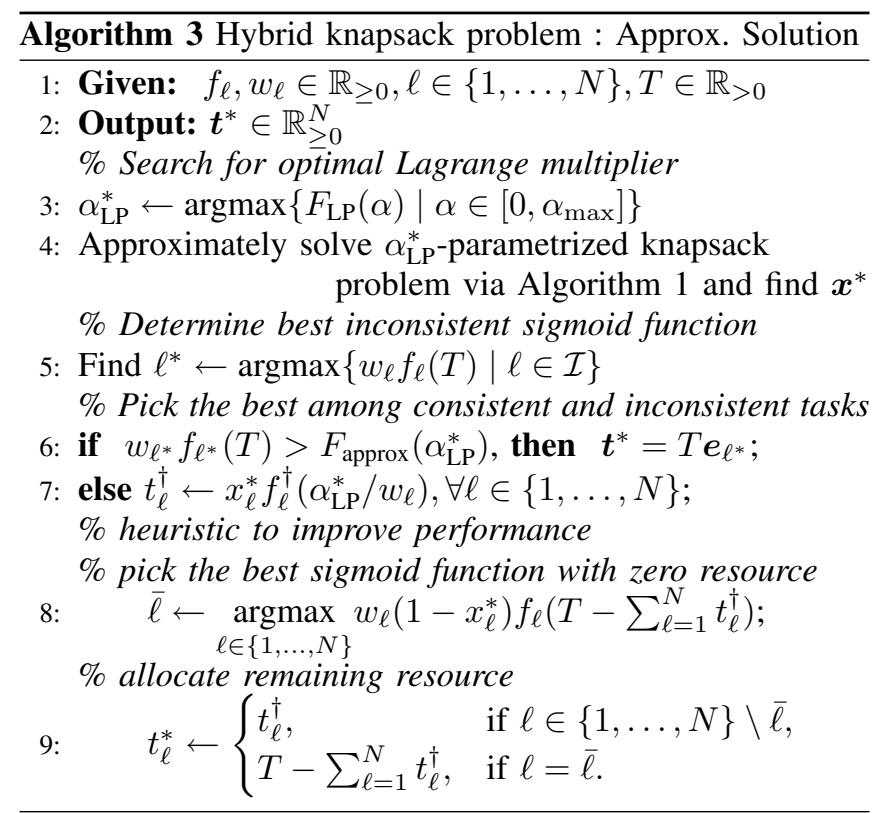

Theorem 4 (Hybrid knapsack problem): The following statements hold for the hybrid knapsack problem (3):

i) The Algorithm 3 provides a solution within factor of optimality 2.

ii) The Algorithm 3 runs in $O\left(N^{2}\right)$ time, provided $F$ is concave on its intervals of continuity.

Proof: We first investigate the properties of the optimal solution. We apply the Karush-Kuhn-Tucker necessary conditions [3] for an optimal solution:

Linear dependence of gradients

$$
\begin{aligned}
& \frac{\partial L}{\partial t_{\ell}^{*}}\left(\boldsymbol{t}^{*}, \alpha^{*}, \boldsymbol{\mu}^{*}\right)=w_{\ell} f_{\ell}^{\prime}\left(t_{\ell}^{*}\right)-\alpha^{*}+\mu_{\ell}^{*}=0, \\
& \forall \ell \in\{1, \ldots, N\} .
\end{aligned}
$$

Feasibility of the solution

$$
\begin{aligned}
T-\mathbf{1}_{N}^{\mathrm{T}} \boldsymbol{t}^{*} & \geq 0 . \\
\boldsymbol{t}^{*} & \succeq 0 .
\end{aligned}
$$

Complementarity conditions

$$
\begin{aligned}
\alpha^{*}\left(T-\mathbf{1}_{N}^{\mathrm{T}} \boldsymbol{t}^{*}\right) & =0 . \\
\mu_{\ell}^{*} t_{\ell}^{*} & =0, \forall \ell \in\{1, \ldots, N\} .
\end{aligned}
$$

Non-negativity of the multipliers

$$
\alpha^{*} \geq 0, \quad \boldsymbol{\mu}^{*} \succeq 0 .
$$

Since $f_{\ell}$ is a strictly increasing function, for each $\ell \in$ $\{1, \ldots, N\}$, the constraint (7) should be active, and thus, from complementarity condition (9) $\alpha^{*}>0$. Further, from equation (10), if $t_{\ell}^{*} \neq 0$, then $\mu_{\ell}^{*}=0$. Therefore, if a non-zero resource is allocated to sigmoid function $f_{\eta}, \eta \in$ $\{1, \ldots, N\}$, then it follows from equation (6)

$$
w_{\eta} f_{\eta}^{\prime}\left(t_{\eta}^{*}\right)=\alpha^{*} \text {. }
$$

Assuming each $f_{\ell}$ is consistent, i.e., $t_{\ell}^{\text {inf }} \leq T$, for each $\ell \in$ $\{1, \ldots, N\}$, the second order condition [14] yields that a local maxima exists at $\boldsymbol{t}^{*}$ only if

$$
f_{\eta}^{\prime \prime}\left(t_{\eta}^{*}\right) \leq 0 \Longleftrightarrow t_{\eta}^{*} \geq t_{\eta}^{\text {inf }}
$$

The equations (12) and (13) yield that optimal non-zero allocation to sigmoid function $f_{\eta}$ is

$$
t_{\eta}^{*}=f_{\eta}^{\dagger}\left(\alpha^{*} / w_{\eta}\right) \text {. }
$$

Given the optimal Lagrange multiplier $\alpha^{*}$, the optimal non-zero allocation to the sigmoid function $f_{\eta}$ is given by equation (14). Further, the optimal set of sigmoid functions with non-zero allocations is the solution to the $\alpha^{*}$ parametrized knapsack problem (4). We now show that $\alpha^{*}$ is maximizer of $F$, and hence, can be searched. Since, at least one task is processed, $w_{\ell} f_{\ell}^{\prime}\left(t_{\ell}^{*}\right)=\alpha$, for some $\ell \in\{1, \ldots, N\}$. Thus, $\alpha \in\left[0, \alpha_{\max }\right]$. By contradiction assume that $\bar{\alpha}$ is the maximizer of $F$, and $F(\bar{\alpha})>F\left(\alpha^{*}\right)$. This means that the allocation corresponding to $\bar{\alpha}$ yields higher reward than the allocation corresponding to $\alpha^{*}$. This contradicts equation (14).

If $t_{\ell}^{\text {inf }}>T$, for some $\ell \in\{1, \ldots, N\}$, then equation (13) does not hold for any $t_{\ell} \in[0, T]$. Since, $f_{\ell}$ is convex in the interval $[0, T]$, the optimal allocation for maximum is at the boundary, i.e., $t_{\ell} \in\{0, T\}$. Therefore, as exemplified in Figure 3, the optimal allocation is either $T \boldsymbol{e}_{\ell}$ or lies at the projection of the simplex on the hyperplane $t_{\ell}=0$. The projection of the simplex on the hyperplane $t_{\ell}=0$ is again a simplex and the argument holds recursively.

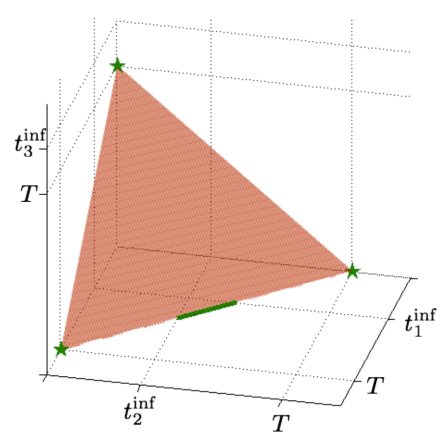

Fig. 3. Possible locations of the maximum are shown in green stars and solid green line. The maximum possible allocation $T$ is smaller than the inflection point of the third sigmoid function. For any allocation to the third sigmoid function, the corresponding entry in the Hessian matrix is positive, and the optimal allocation to third sigmoid function is 0 or $T$. Optimal allocation to the first and second sigmoid function may lie at the vertex of simplex, or at a location where Jacobian is zero and Hessian matrix is negative definite.

Since $\alpha_{\mathrm{LP}}^{*}$ is maximizer of $F_{\mathrm{LP}}$, and the $\alpha$-parametrized fractional knapsack problem is relaxation of $\alpha$-parametrized knapsack problem, hence

$$
F_{\mathrm{LP}}\left(\alpha_{\mathrm{LP}}^{*}\right) \geq F_{\mathrm{LP}}\left(\alpha^{*}\right) \geq F\left(\alpha^{*}\right) .
$$

Also, $\alpha^{*}$ is maximizer of $F$ and $F_{\text {approx }}$ is sub-optimal value 
of the objective function, hence

$$
F\left(\alpha^{*}\right) \geq F\left(\alpha_{\mathrm{LP}}^{*}\right) \geq F_{\text {approx }}\left(\alpha_{\mathrm{LP}}^{*}\right) \geq \frac{1}{2} F_{\mathrm{LP}}\left(\alpha_{\mathrm{LP}}^{*}\right),
$$

where the last inequality follows from the standard proof [13] of the fact that Algorithm 1 is optimal within factor of optimality 2. The value of objective function at allocation $\boldsymbol{t}^{\dagger}$ in Algorithm 3 is equal to $F_{\text {approx }}\left(\alpha_{\mathrm{LP}}^{*}\right)$. The allocation $\boldsymbol{t}^{\dagger}$ may not saturate the total available resource $T$. Since, the sigmoid functions are increasing function of the allocated resource, the total resource must be utilized, and it is heuristically done in step 8 and 9 of the Algorithm 3. This improves the value of the objective function and the factor of optimality remains at most 2. This establishes the first statement.

To prove the second statement, we note that if $F$ is concave on its intervals of continuity, then the maximum over each interval can be searched through bisection method in fixed number of steps. It follows from Lemma 3 that the maximum number of intervals is $N+1$. Further, each step of the bisection method involves the solution of $\alpha$-parametrized fractional knapsack problem, which can be computed in $O(N)$ time. Thus, the Algorithm 3 runs in $O\left(N^{2}\right)$ time and this completes the proof of the theorem.

Corollary 5 (Identical sigmoid functions): Given identical sigmoid functions $f$ and weights in hybrid knapsack problem (3) the optimal solution $\boldsymbol{t}^{*}$ is an $N$-tuple with $m^{*}$ entries equal to $T / m^{*}$ and all other entries zero, where

$$
m^{*}=\underset{m \in\{1, \ldots, N\}}{\operatorname{argmax}} m f(T / m) .
$$

Proof: It follows from equation (14) that, for identical sigmoid functions, the optimal non-zero resource allocated is the same for each sigmoid function. The number of sigmoid functions with optimal non-zero resource is determined by equation (17), and the statement follows.

Example 6: Given sigmoid functions $f_{\ell}(t)=1 /(1+$ $\left.\exp \left(-a_{\ell} t+b_{\ell}\right)\right), \ell \in\{1, \ldots, 10\}$ with parameters and associated weights

$$
\begin{aligned}
\boldsymbol{a} & =\left(a_{1}, \ldots, a_{10}\right)=(1,2,1,3,2,4,1,5,3,6), \\
\boldsymbol{b} & =\left(b_{1}, \ldots, b_{10}\right)=(5,10,3,9,8,16,6,30,6,12), \\
\text { and } \boldsymbol{w} & =\left(w_{1}, \ldots, w_{10}\right)=(2,5,7,4,9,3,5,10,13,6),
\end{aligned}
$$

and total resource $T=15$ units. The optimal solution and the approximate solution without the heuristic in step 8 and 9 of the Algorithm 3 are shown in Figure 4. The approximate solution with the performance improvement heuristic in step 8 and 9 of the Algorithm 3 gives the same solution as the optimal solution. The functions $F, F_{\text {approx }}$, and $F_{\mathrm{LP}}$ are shown in Figure 5.

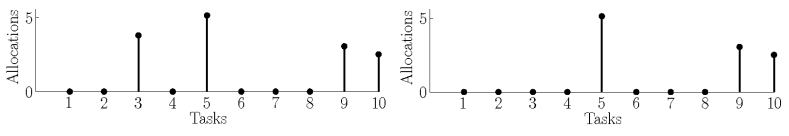

Fig. 4. Optimal allocations and the approximate optimal allocations without performance improvement heuristic.

\section{Hybrid BIN-PACKING PROBLEM}

\section{A. Problem description}

Consider $N$ sigmoid functions $f_{\ell}, \ell \in\{1, \ldots, N\}$, and resource $T \in \mathbb{R}_{>0}$. Determine the minimum $K \in \mathbb{N}$ and a mapping $\Upsilon:\{1, \ldots, N\} \rightarrow\{1, \ldots, K\}$ such that, for each

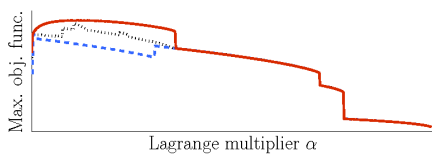

Fig. 5. Exact and approximate maximum value of the objective function The functions $F_{\mathrm{LP}}, F, F_{\text {approx }}$ are shown by solid brown line, black dotted line, and blue dashed line, respectively. The points of discontinuity of function $F_{\mathrm{LP}}$ are at points where the Lagrange multiplier has value in the set $\left\{\alpha_{1}, \ldots, \alpha_{N}\right\}$.

$i \in\{1, \ldots, K\}$, the optimal solution to the hybrid knapsack problem

$$
\begin{array}{ll}
\operatorname{maximize} & \sum_{\ell \in \mathcal{A}_{i}} f_{\ell}\left(t_{\ell}\right) \\
\text { subject to } & \sum_{\ell \in \mathcal{A}_{i}} t_{\ell}=T,
\end{array}
$$

where $\mathcal{A}_{i}=\{j \in\{1, \ldots, N\} \mid \Upsilon(j)=i\}$, allocates nonzero resource to each sigmoid function $f_{\ell}, \ell \in \mathcal{A}_{i}$. Note that the Algorithm 3 provides the exact solution to hybrid knapsack problem when each task is processed.

The hybrid bin-packing problem models a situation where one needs to determine the minimum number of operators, each working for time $T$, required to optimally serve each of the $N$ tasks characterized by functions $f_{\ell}, \ell \in\{1, \ldots, N\}$.

\section{B. Optimal solution}

An approximation algorithm to solve hybrid bin-packing problem is presented in Algorithm 4. This algorithm is similar to the standard next-fit algorithm and adds a sigmoid function to a bin if optimal policy allocates non-zero resource to each sigmoid function. Otherwise, it opens a new bin. We now present a formal analysis of this algorithm. We introduce following notations. Let $K^{*}$ be the optimal solution of the hybrid bin-packing problem, and $K_{\text {next-fit }}$ be the solution obtained through Algorithm 4. We denote the critical penalty rate for sigmoid function $f_{\ell}$ by $\psi_{\ell}, \ell \in\{1, \ldots, N\}$, and let $\psi_{\min }=\min \left\{\psi_{\ell} \mid \ell \in\{1, \ldots, N\}\right\}$. Before we analyze Algorithm 4, we present the following important property of optimization problem (18).

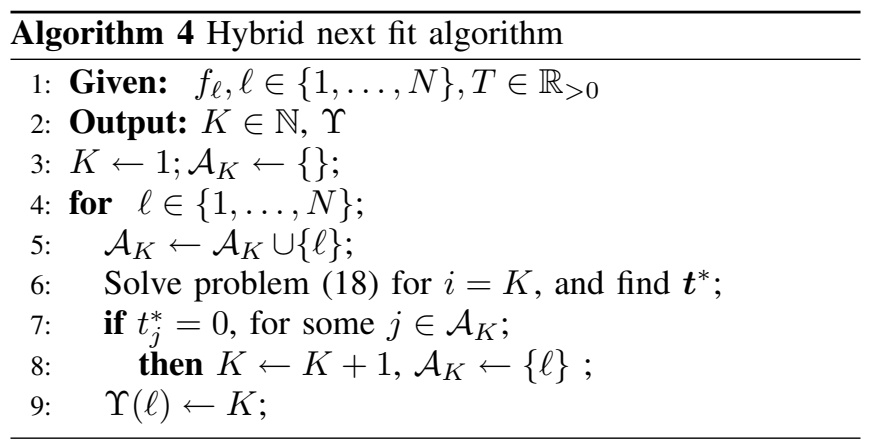

Lemma 7 (Non-zero allocations): A solution to the optimization problem (18) allocates non-zero resource to sigmoid function $f_{\ell}$, for each $\ell \in \mathcal{A}_{i}, i \in\{1, \ldots, K\}$, if

$$
T \geq \sum_{\ell \in \mathcal{A}_{i}} f_{\ell}^{\dagger}\left(\psi_{\min }\right)
$$

Proof: It suffices to prove that if $T=\sum_{\ell \in \mathcal{A}_{i}} f_{\ell}^{\dagger}\left(\psi_{\text {min }}\right)$, then $\psi_{\min }$ is the optimal Lagrange multiplier $\alpha^{*}$ in Algorithm 3. Since, $t_{\ell}^{*}=f_{\ell}^{\dagger}\left(\psi_{\text {min }}\right), \ell \in \mathcal{A}_{i}$ are feasible nonzero allocations, $\psi_{\min }$ is a Lagrange multiplier. We now prove that $\psi_{\min }$ is the optimal Lagrange multiplier. Let 
$\mathcal{A}_{i}=\left\{1, \ldots, a_{i}\right\}$. By contradiction, assume that $\boldsymbol{t}^{*}$ is not the globally optimal allocation. Without loss of generality, we assume that the global policy allocates zero resource to sigmoid function $f_{a_{i}}$, and $\bar{t}$ be the globally optimal allocation. We observe that

$$
\begin{aligned}
& \sum_{\ell=1}^{a_{i}-1} f_{\ell}\left(\bar{t}_{\ell}\right)+f_{a_{i}}(0) \leq \sum_{\ell=1}^{a_{i}-1} f_{\ell}\left(\bar{t}_{\ell}\right)+f_{a_{i}}\left(t_{a_{i}}^{*}\right)-\psi_{\min } t_{a_{i}}^{*} \\
& \leq \sum_{\ell=1}^{a_{i}} f_{\ell}\left(t_{\ell}^{*}\right)+\sum_{\ell=1}^{a_{i}-1} f_{\ell}^{\prime}\left(t_{\ell}^{*}\right)\left(\bar{t}_{\ell}-t_{\ell}^{*}\right)-\psi_{\min } t_{a_{i}}^{*}=\sum_{\ell=1}^{a_{i}} f_{\ell}\left(t_{\ell}^{*}\right),
\end{aligned}
$$

where inequalities follow from the definition of critical penalty and the concavity to the sigmoid function at $t_{\ell}^{*}$, respectively. This contradicts our assumption. Hence, $t^{*}$ is the global optimal allocation.

Theorem 8 (Hybrid bin-packing problem): The following statements hold for the hybrid bin-packing problem:

i) The optimal solution satisfies the following bounds

$$
K_{\text {next-fit }} \geq K^{*} \geq \frac{1}{T} \sum_{\ell=1}^{N} \min \left\{T, t_{\ell}^{\text {inf }}\right\} .
$$

ii) The solution obtained through Algorithm 4 satisfies

$$
K_{\text {next-fit }} \leq \frac{1}{T}\left(2 \sum_{\ell=1}^{N} f_{\ell}^{\dagger}\left(\psi_{\min }\right)-1\right) .
$$

iii) The Algorithm 4 provides a solution to the hybrid binpacking problem within a factor of optimality

$$
\frac{\max \left\{2 f_{\ell}^{\dagger}\left(\psi_{\text {min }}\right) \mid \ell \in\{1, \ldots, N\}\right\}}{\max \left\{\min \left\{T, t_{\ell}^{\text {inf }}\right\} \mid \ell \in\{1, \ldots, N\}\right\}} .
$$

Proof: It follows from Algorithm 3 that if $t_{\ell}^{\text {inf }}<T$, then the optimal non-zero allocation to sigmoid function $f_{\ell}$ is greater than $t_{\ell}^{\text {inf }}$. Otherwise, the optimal non-zero allocation is equal to $T$. Therefore, if each sigmoid function gets a non-zero allocation under the optimal policy, then at least $\sum_{\ell=1}^{N} \min \left\{T, t_{\ell}^{\mathrm{inf}}\right\}$ resource is required, and the lower bound on the optimal $K^{*}$ follows.

It follows from Lemma 7 that if resource $t_{\ell}=f_{\ell}^{\dagger}\left(\psi_{\min }\right)$ is available for each task $\ell \in\{1, \ldots, N\}$, then a nonzero resource is allocated to it. Therefore, the solution of the bin-packing problem with bin size $T$ and items of size $\left\{f_{\ell}^{\dagger}\left(\psi_{\min }\right) \mid \ell \in\{1, \ldots, N\}\right\}$ provides an upper bound to the solution of the hybrid bin-packing problem. The upper bound to the solution of this bin-packing problem obtained through the next-fit algorithm 2 is $\left(2 \sum_{\ell=1}^{N} f_{\ell}^{\dagger}\left(\psi_{\min }\right)-1\right) / T$, and this completes the proof of the second statement.

The third statement follows immediately from the first two statements.

Example 9: For the same set of sigmoid functions as in Example 6 and $T=20$ units, the solution to the hybrid binpacking problem obtained through hybrid next fit algorithm requires $K_{\text {next-fit }}=3$ bins, and the optimal allocations to each task in these bins are shown in Figure 6.

\section{CONCLUSiOnS AND Future DiRections}

We studied non-convex hybrid optimization problems involving sigmoid functions. We considered the maximization of a sigmoid function subject to a linear penalty and showed that the optimal allocations jumps down to zero at a critical

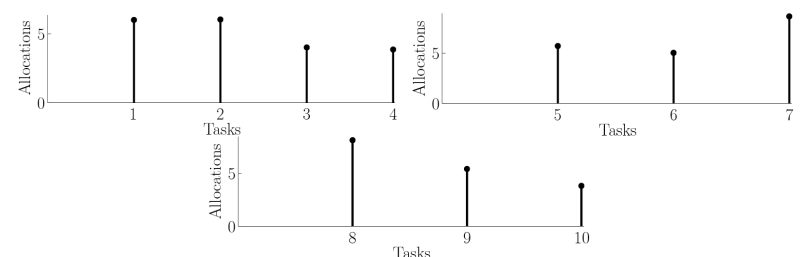

Fig. 6. Allocations to sigmoid functions in each bin.

penalty rate. This jump in the allocation imparts combinatorial effects to the constrained optimization problems involving sigmoid functions. We studied two such problems, namely, hybrid knapsack problem and hybrid bin-packing problem. We utilized the approximation algorithms for the standard knapsack problem and the bin-packing problem to develop approximation algorithms for these problems.

There are many possible extensions of this work. A similar strategy for approximate optimization could be adopted for other problems involving sigmoid functions, e.g., the network utility maximization problem. Other extensions include problems involving general non-convex functions and optimization in queues with sigmoid characteristics.

\section{REFERENCES}

[1] S. Ağrali and J. Geunes. Solving knapsack problems with Scurve return functions. European Journal of Operational Research, 193(2):605-615, 2009

[2] R. Bogacz, E. Brown, J. Moehlis, P. Holmes, and J. D. Cohen. The physics of optimal decision making: A formal analysis of performance in two-alternative forced choice tasks. Psychological Review, 113(4):700-765, 2006.

[3] S. Boyd and L. Vandenberghe. Convex Optimization. Cambridge University Press, 2004.

[4] K. M. Bretthauer and B. Shetty. The nonlinear knapsack problemalgorithms and applications. European Journal of Operational Research, 138(3):459-472, 2002.

[5] W. M. Bulkeley. Chicago's camera network is everywhere. The Wall Street Journal, Nov 172009.

[6] G. J. Burke, J. Geunes, H. E. Romeijn, and A. Vakharia. Allocating procurement to capacitated suppliers with concave quantity discounts. Operations Research Letters, 36(1):103-109, 2008.

[7] M. Fazel and M. Chiang. Network utility maximization with nonconcave utilities using sum-of-squares method. In IEEE Conf. on Decision and Control and European Control Conference, pages 18671874, Seville, Spain, December 2005.

[8] J. R. Freeland and C. B. Weinberg. S-Shaped response functions: Implications for decision models. Journal of the Operational Research Society, 31(11):1001-1007, 1980.

[9] W. Ginsberg. The multiplant firm with increasing returns to scale. Journal of Economic Theory, 9(3):283-292, 1974.

[10] T. Ibaraki and N. Katoh. Resource Allocation Problems: Algorithmic Approaches. MIT Press, 1988.

[11] S. Kameshwaran and Y. Narahari. Nonconvex piecewise linear knapsack problems. European Journal of Operational Research, 192(1):5668, 2009.

[12] H. Kellerer, U. Pferschy, and D. Pisinger. Knapsack Problems. Springer, 2004.

[13] B. Korte and J. Vygen. Combinatorial Optimization: Theory and Algorithms, volume 21 of Algorithmics and Combinatorics. Springer, 4 edition, 2007.

[14] D. G. Luenberger. Linear and Nonlinear Programming. AddisonWesley, 2 edition, 1984.

[15] J. J. Moré and S. A. Vavasis. On the solution of concave knapsack problems. Mathematical programming, 49(1):397-411, 1990.

[16] R. W. Pew. The speed-accuracy operating characteristic. Acta Psychologica, 30:16-26, 1969.

[17] T. Shanker and M. Richtel. In new military, data overload can be deadly. The New York Times, Jan 16, 2011.

[18] V. Srivastava, R. Carli, F. Bullo, and C. Langbort. Task release control for decision making queues. In American Control Conference, pages 1855-1860, San Francisco, CA, USA, June 2011. 